\title{
A Multi-Stage Advanced Deep Learning Graphics Pipeline
}

\author{
Mark Wesley Harris \\ wharris2@uccs.edu \\ University of Colorado at Colorado Springs \\ Colorado Springs, Colorado, USA
}

\author{
Sudhanshu Kumar Semwal \\ ssemwal@uccs.edu \\ University of Colorado at Colorado Springs \\ Colorado Springs, Colorado, USA
}

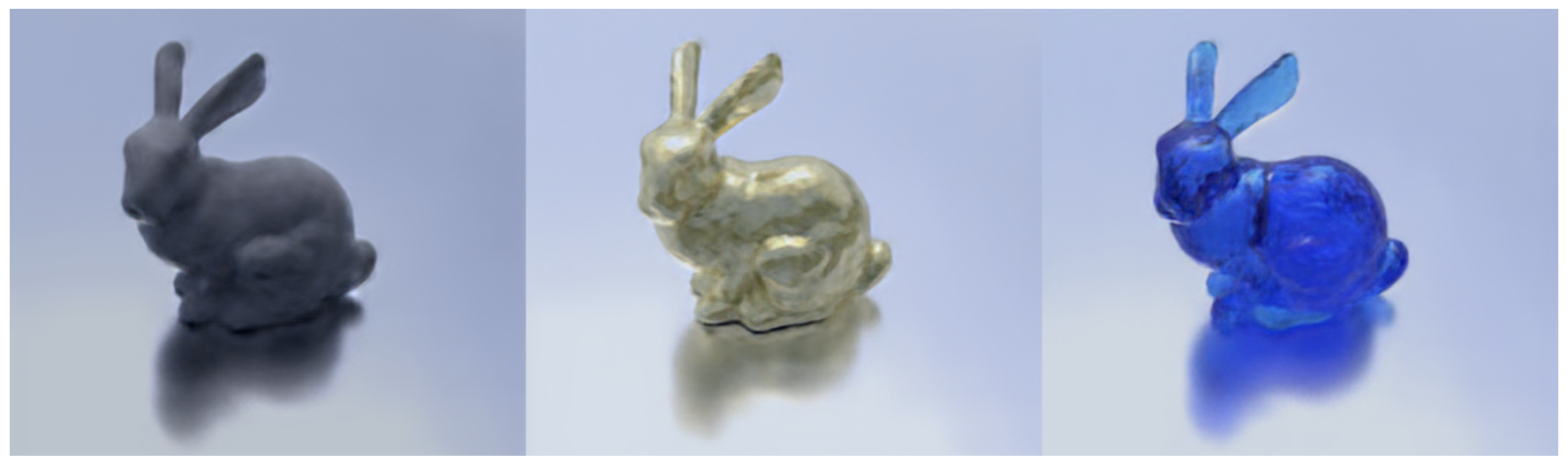

Figure 1: Final renders from the proposed Advanced Deep Learning Graphics Pipeline. 18,225 input attribute files were used to generate each image. Bunny model courtesy of the Stanford University 3D Scanning Repository [Turk and Levoy 1994].

\begin{abstract}
In this paper we propose the Advanced Deep Learning Graphics Pipeline (ADLGP). ADLGP is a novel approach that uses existing deep learning architectures to convert scene data into rendered images. Our goal of generating frames from semantic data has produced successful renderings with similar structures and composition as target frames. We demonstrate the success of ADLGP with side-by-side comparisons of frames generated through standard rendering procedures. We assert that a fully implemented ADLGP framework would reduce the time spent in visualizing 3D environments, and help selectively offload the requirements of the current graphics rendering pipeline.
\end{abstract}

\section{CCS CONCEPTS}

- Computing methodologies $\rightarrow$ Rendering; Image processing; Machine learning algorithms.

\section{KEYWORDS}

Graphics pipeline, rendering pipeline, rendering technologies, machine learning, generative adversarial networks, text-to-image, super resolution, semantic data processing, image processing

This work is licensed under a Creative Commons Attribution-NonCommercial International 4.0 License.

SA '21 Technical Communications, December 14-17, 2021, Tokyo, Japan (C) 2021 Copyright held by the owner/author(s)

ACM ISBN 978-1-4503-9073-6/21/12.

https://doi.org/10.1145/3478512.3488609
ACM Reference Format:

Mark Wesley Harris and Sudhanshu Kumar Semwal. 2021. A Multi-Stage Advanced Deep Learning Graphics Pipeline. In SIGGRAPH Asia 2021 Technical Communications (SA '21 Technical Communications), December 14-17, 2021, Tokyo, Japan. ACM, New York, NY, USA, 4 pages. https://doi.org/10. $1145 / 3478512.3488609$

\section{INTRODUCTION}

Our goal is to train a framework of deep neural networks to learn the correspondence between semantic data within an animated 3D environment and rendered target frames. We propose the application of the Advanced Deep Learning Graphics Pipeline (ADLGP) to offload existing graphics rendering so that future visual experiences, such as 3D games, are more interactive. The system design displayed in Figure 2 shows how ADLGP could be used to supplement current graphics rendering with generated frames using trained machine learning architectures, thereby lowering the time and costs incurred by complex rendering calculations. In Figure 5 we present a total of six frames generated by our ADLGP framework, which was trained on the first and third frames of a source animation. Generated frames 2 and 4 through 6 are novel views with respect to the dataset. Our results show that ADLGP can be used to generate quality in-between frames and also predict plausible future frames, given they have the same dimensional features as those trained on. In a full implementation of our work, training would be dynamic; the framework would use frames generated from the standard graphics pipeline to dynamically train the generative models of the framework, and then use ADLGP to replace strategic future frames when low enough loss has been achieved.

\section{RELATED WORK}

Path tracing [Foley et al. 2013] is very computationally slow [Schied et al. 2017], however efforts using neural networks to improve 


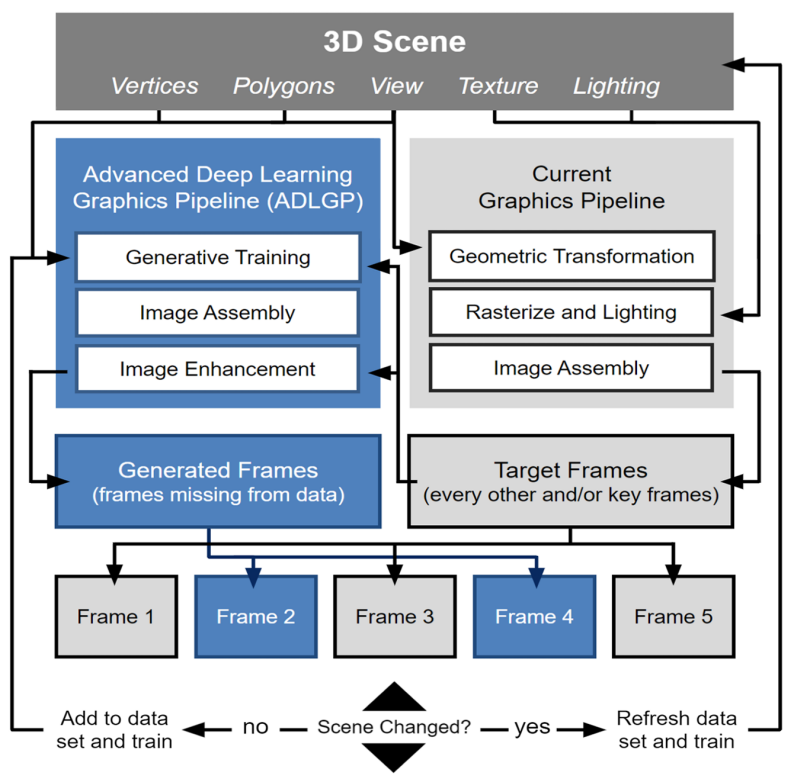

Figure 2: Process diagram for a real-time application of the Advanced Deep Learning Graphics Pipeline framework.

image quality and rendering time have been gaining ground over the last few years. Image-based approaches [Chaitanya et al. 2017; Schied et al. 2017], and convolutional LSTMs [Mahjourian et al. 2017] for predicting frames are a few examples. Mathieu et al. use a multi-scale GAN approach [Mathieu et al. 2015] to address the major problems of exemplar prediction - lower output quality as convolutions may create blurry predictions. The convolutional encoder-decoder network, Deep Voxel Flow, borrows voxels from neighboring frames [Liu et al. 2017] requiring less processing than other image-to-image methods. Qiao et al. proposed improvements to the Attentional GAN model [Xu et al. 2018] with the LEarn, Imagine and CreAte GAN (LeicaGAN) [Qiao et al. 2019a]. Hong et al. created an intermediate semantic layout to further improve regional influence [Hong et al. 2018; Li et al. 2019], yet poor object prediction disqualifies it for use in novel image generation in our application.

Many architectures have also been created for the purpose of enhancing low quality images via super resolution. Dahl et al. at Google Brain extended PixelCNN [Oord et al. 2016] to address the problem of recursive super resolution [Dahl et al. 2017]. The SuperResolution GAN (SRGAN) successfully translates between low and high resolution images [Goodfellow et al. 2016; Ledig et al. 2017] Ledig et al. showed that their perceptual loss greatly improved the ability for the SRGAN model to create high resolution outputs of input images with improved perceived quality over models without perceptual loss [Ledig et al. 2017; Simonyan and Zisserman 2015; Zhou et al. 2018].

\section{PROPOSED RESEARCH}

Our work is based on the application of deep learning networks to the rendering process [Harris and Semwal 2021]. We use solely non-image semantic data extracted from the scene in order to create images, the same inputs consumed in graphics rendering. We chose to use a sequence-to-sequence learning mechanism called attention, which follows an encoder-decoder structure similar to other sequence-to-sequence learning techniques, yet is better suited for longer sequences of data. Recently, attention was applied in conditional GANs for the purpose of improving text-to-image translation [Vaswani et al. 2017]. The AttnGAN model combines adversarial learning and attention to improve attribute correspondence and semantic relevance to input descriptors [Xu et al. 2018]. The first stage of ADLGP uses AttnGAN to convert 3D scene data in the form of semantic text attributes into a structural image representing a low resolution rendering of the scene. To improve further on the results of [Harris and Semwal 2021], we add a multi-stage framework. Muti-stage frameworks have been used by several other researchers to generate high-quality images with large resolutions ([Mathieu et al. 2015], [Karnewar et al. 2019], [Qiao et al. 2019b], [Li et al. 2019], and [Qiao et al. 2019a]). Using the SRGAN super resolution architecture [Ledig et al. 2017] trained on the structural outputs of the AttnGAN [Xu et al. 2018], we obtained significant increases in quality for predicting known and unknown views. We also tested the capabilities of ADLGP in generating different material types, including properties of diffuse, reflected, and refracted light (see Figure 5). The final ADLGP framework was used to generate realistic frame sequences for all of these materials.

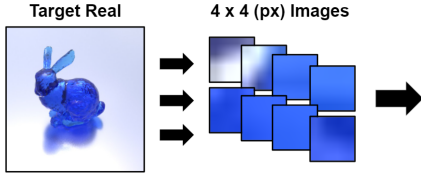

1. Generate dataset and remove duplicates
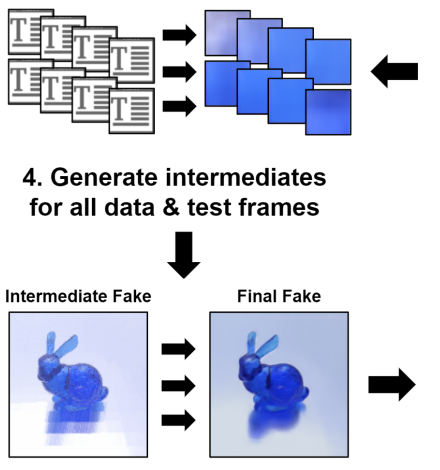

5. Train SRGAN to improve image quality

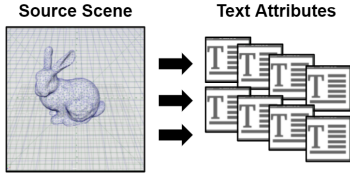

2. Generate attributes from Maya source file

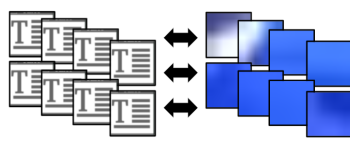

3. Train AttnGAN text-to-image generation

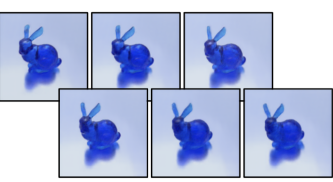

6. Render final sequence of train \& test frames
Figure 3: Flow diagram of ADLGP, as applied in our evaluation. Bunny model courtesy of the Stanford University 3D Scanning Repository [Turk and Levoy 1994].

\section{ADLGP IMPLEMENTATION}

Our process is illustrated in Figure 3. We generated sets of training data by extracting image-text pairs from the source scene. Using 


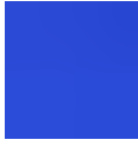

Floor.d:3974854 and Floor.i:4056 also UpperBo dy.d:4236229 and UpperBody.i:4188 and UpperBo dy.v: 65535 and UpperBody.r:0-0-0 also BackRig htLeg.d:4236229 and BackRightLeg.i:4189 and BackRightLeg.v: 65535 and BackRightLeg.r:0-0-0

Figure 4: Sample $4 \times 4$ pixel image and corresponding text attributes extracted from the scene.

our novel image generation process [Harris and Semwal 2021], semantic text attributes are extracted from the animation software for every frameblock of the frame. A frameblock is a small portion of pixels which show significant change in the animation. An example of a text-image pair is displayed in Figure 4.

We used the Stanford Bunny [Turk and Levoy 1994] to evaluate the capabilities of our proposed system. We trained the AttnGAN pre-training and generative models, which were used to generate structural images off of the extracted text. The AttnGAN rendered adequate experimental images, however many aspects of the architecture limited the capacity of understanding scene attributes. A major drawback of AttnGAN is its limitations for recognizing the inherent semantic dependencies within data, such as the closeness of vector components. Semantic attributes which helped the most in generating good results were merely identifiers. In this implementation, we identified where in a frameblock an object resided using subdivision and clipping, as well as what part of the object was shown by grouping visible faces together. Because the sets of these values are not correlated to block location, they greatly improved recognition for the AttnGAN model overall. We found the use of these identifiers impacted our results more so than any numerical attributes. To improve recognition for numerical values, such as rotation, translation, and scale, the generative model used in ADLGP must be better equipped to handle such inherent relationships.

Once the AttnGAN model was trained, we created a dataset of intermediate frames to use in training the SRGAN model. To create an intermediate frame from outputs of AttnGAN, semantic attributes of the frame were used to generate all frameblocks which were then placed into their corresponding block positions of each frame. This gave us the structural base used to train and test the SRGAN model. Because SRGAN is trained on intermediate outputs, all known training frames must be generated and trained on to completely initialize the network. Any frame generated by AttnGAN can then be fed into the initialized SRGAN model to output the final enhanced image. Retraining is only required for large changes between frames, such as changes in scene. This process was completed for the sequence of the 6 frames we evaluated, only 2 of which were known to the training dataset.

\section{RESULTS}

Our final results were generated from $540 \times 540$ pixel resolution frames using $4 \times 4$ pixel dimension frameblocks. Our outputs are visually very promising, as shown in Figure 5. To compare the results of the architecture between those from frame prediction and other baselines, we decided to measure differences between our outputs and the target images. A summary of these results are shown in Table 1. The AttnGAN architecture trained for 100 epochs on each of our datasets, which contained approximately 24,000 training images. The SRGAN model was trained on the resulting 6 frames for 2000 epochs. Given the lack of training data compared to the case of AttnGAN, we found a higher number of epochs was necessary for convergence of the SRGAN architecture.

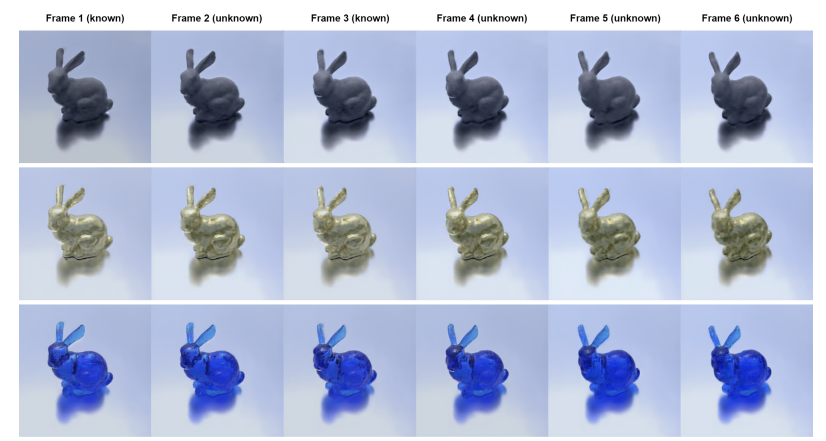

Figure 5: Sequence of six frames rendered with ADLGP for clay, gold, and glass materials. Bunny model courtesy of the Stanford University 3D Scanning Repository [Turk and Levoy 1994].

Our final results are shown in Figure 5. All images are structurally very similar to their targets. The architecture worked well for inbetween frames, where the attributes of the frame were closer to those of known data. When these attributes were not highly correlated, rendering of the frame was less sharp, as in frames 5 and 6 generated by ADLGP. The closeness of generated and target frames both visually and numerically is very promising for future work in applying deep learning to the graphics pipeline, and shows the promise of ADLGP for rendering novel views of a scene.

During testing of ADLGP, We observed that extremely fine lighting and textural details were often incorrect. This is in large part due to the absence of textural and lighting information in our attributes. Future improvements to these qualities would improve ADLGP renderings. While the SRGAN model smoothed over clean edges, overall visual quality was greatly improved by the addition of the super resolution phase. In a subjective comparison, our ADLGP framework came close to or surpassed results for current frame prediction models. The Geometry-Based Next Frame Prediction model estimated scores between 24 and 25 PSNR and 0.92 and 0.93 SSIM, on average [Mahjourian et al. 2017]. Our results for PSNR exceed those ranges for most cases. The SSIM values for our highest scoring model neared 0.97 with an average value of 0.948 , calculated from Table 1. SSIM and PSNR were also improved for our model when compared to STP [Finn et al. 2016] and Multi-Scale Video Prediction [Mathieu et al. 2015]. We concede there may be situations where our proposed framework performs more poorly than we recorded in our experiments. Regardless, our comparisons with current frame prediction networks show that our method has major promise and requires broader studies in future work [Zhou et al. 2020]. 
Table 1: Averaged results of MSE, SSIM and PSNR for intermediate and enhanced outputs. The best value of each column is shown in bold.

\begin{tabular}{|cccc|}
\hline \multicolumn{4}{c|}{ Intermediate Outputs } \\
\hline Material & MSE & SSIM & PSNR \\
\hline Clay & 28.847 & $\mathbf{0 . 9 0 4}$ & 33.532 \\
\hline Gold & $\mathbf{2 6 . 4 0 9}$ & 0.876 & $\mathbf{3 3 . 9 1 9}$ \\
\hline Glass & 29.962 & 0.874 \\
\hline \multicolumn{4}{c|}{ Enhanced Outputs } \\
\hline Material & MSE & SSIM \\
\hline Clay & 111.231 & 0.951 & PSNR \\
\hline Gold & $\mathbf{1 1 0 . 0 0 8}$ & $\mathbf{0 . 9 5 3}$ & 27.675 \\
\hline Glass & 112.110 & 0.942 & 22.998 \\
\hline
\end{tabular}

\section{CONCLUSIONS AND FUTURE RESEARCH}

In this paper, we studied the semantics available in the scene and exported the semantic information as textual attributes. Our novel approach of breaking the viewport into frameblocks greatly improved rendering results and reduced the chances of over-training. We explored various avenues of text-to-image translation, and described works from the related field of image-to-image frame prediction. We found that a multi-stage approach is preferable for complex image generation in most cases, where a structural image is first created and then later refined to generate the final enhanced output. Using the existing Attentional GAN [Xu et al. 2018] and Super-Resolution GAN [Ledig et al. 2017] architectures, we successfully implemented an Advanced Deep Learning Graphics Pipeline. Once trained, the pipeline was able to generate plausible novel frames without a commercial renderer. Evaluating our final results using MSE, SSIM, and PSNR, we found our approach to show great promise compared to current frame prediction strategies.

As the quality of ADLGP generated images in our paper are promising, we expect ADLGP to be implemented in the future alongside the standard graphics pipeline, as shown in Figure 2, to leverage the speed of ADLGP and remove barriers of computation inherent to the rendering process.

\section{ACKNOWLEDGMENTS}

We gratefully acknowledge the SRGAN and AttnGAN communities and the Stanford University 3D Scanning Repository for the materials which were used to develop our thesis work, algorithms, and analysis in this paper.

\section{REFERENCES}

Chakravarty R. Alla Chaitanya, Anton S. Kaplanyan, Christoph Schied, Marco Salvi, Aaron Lefohn, Derek Nowrouzezahrai, and Timo Aila. 2017. Interactive Reconstruction of Monte Carlo Image Sequences Using a Recurrent Denoising $\mathrm{Au}$ toencoder. ACM Trans. Graph. 36, 4, Article 98 (July 2017), 12 pages. https: //doi.org/10.1145/3072959.3073601

R. Dahl, M. Norouzi, and J. Shlens. 2017. Pixel Recursive Super Resolution. In 2017 IEEE International Conference on Computer Vision (ICCV). 5449-5458. https://doi. org/10.1109/ICCV.2017.581

Chelsea Finn, Ian Goodfellow, and Sergey Levine. 2016. Unsupervised Learning for Physical Interaction through Video Prediction. In Proceedings of the 30th International Conference on Neural Information Processing Systems (Barcelona, Spain) (NIPS'16). Curran Associates Inc., Red Hook, NY, USA, 64-72.
James D. Foley, Andries van Dam, Steven K. Feiner, and John F. Hughes. 2013. Computer Graphics: Principles and Practice (3rd Ed.). Addison-Wesley Longman Publishing Co., Inc., Boston, MA, USA.

Ian Goodfellow, Yoshua Bengio, and Aaron Courville. 2016. Deep Learning. The MIT Press.

Mark Wesley Harris and Sudhanshu Kumar Semwal. 2021. Deep Rendering Graphics Pipeline. Computer Science Research Notes - CSRN CSRN 3101 (May 2021), 101-108. https://doi.org/10.24132/CSRN.2021.3101.11

S. Hong, D. Yang, J. Choi, and H. Lee. 2018. Inferring Semantic Layout for Hierarchical Text-to-Image Synthesis, In 2018 IEEE/CVF Conference on Computer Vision and Pattern Recognition. CVPR, 7986-7994. https://doi.org/10.1109/CVPR.2018.00833

Animesh Karnewar, Oliver Wang, and Raghu Sesha Iyengar. 2019. MSG-GAN: MultiScale Gradient GAN for Stable Image Synthesis. arXiv preprint arXiv:1903.06048 (2019).

Christian Ledig, Lucas Theis, Ferenc Huszar, Jose Caballero, Andrew Cunningham, Alejandro Acosta, Andrew Aitken, Alykhan Tejani, Johannes Totz, Zehan Wang, and Wenzhe Shi. 2017. Photo-Realistic Single Image Super-Resolution Using a Generative Adversarial Network. CVPR, 105-114. https://doi.org/10.1109/CVPR. 2017.19

Wenbo Li, Pengchuan Zhang, Lei Zhang, Qiuyuan Huang, Xiaodong He, Siwei Lyu, and Jianfeng Gao. 2019. Object-driven Text-to-Image Synthesis via Adversarial Training. CoRR abs/1902.10740 (2019). arXiv:1902.10740 http://arxiv.org/abs/1902.10740

Ziwei Liu, Raymond A. Yeh, Xiaoou Tang, Yiming Liu, and Aseem Agarwala. 2017. Video Frame Synthesis Using Deep Voxel Flow. In Proceedings of the IEEE International Conference on Computer Vision (ICCV).

Reza Mahjourian, Martin Wicke, and Anelia Angelova. 2017. Geometry-based next frame prediction from monocular video. In Intelligent Vehicles Symposium (IV), 2017 IEEE. IEEE, 1700-1707.

Michael Mathieu, Camille Couprie, and Yann LeCun. 2015. Deep multi-scale video prediction beyond mean square error. CoRR abs/1511.05440 (2015).

Aäron van den Oord, Nal Kalchbrenner, Oriol Vinyals, Lasse Espeholt, Alex Graves, and Koray Kavukcuoglu. 2016. Conditional Image Generation with PixelCNN Decoders. In Proceedings of the 30th International Conference on Neural Information Processing Systems (Barcelona, Spain) (NIPS'16). Curran Associates Inc., USA, 47974805. http://dl.acm.org/citation.cfm?id=3157382.3157633

Tingting Qiao, Jing Zhang, Duanqing Xu, and Dacheng Tao. 2019a. Learn, Imagine and Create: Text-to-Image Generation from Prior Knowledge. In $A d$ vances in Neural Information Processing Systems 32, H. Wallach, H. Larochelle, A. Beygelzimer, F. Alche-Buc, E. Fox, and R. Garnett (Eds.). Curran Associates, Inc., 887-897. http://papers.nips.cc/paper/8375-learn-imagine-and-create-to-imagegeneration-from-prior-knowledge.pdf

Tingting Qiao, Jing Zhang, Duanqing Xu, and Dacheng Tao. 2019b. MirrorGAN: Learning Text-to-image Generation by Redescription. CoRR abs/1903.05854 (2019). arXiv:1903.05854 http://arxiv.org/abs/1903.05854

Christoph Schied, Anton Kaplanyan, Chris Wyman, Anjul Patney, Chakravarty R. Alla Chaitanya, John Burgess, Shiqiu Liu, Carsten Dachsbacher, Aaron Lefohn, and Marco Salvi. 2017. Spatiotemporal Variance-guided Filtering: Real-time Reconstruction for Path-traced Global Illumination. In Proceedings of High Performance Graphics (HPG '17). ACM, Article 2, 12 pages. https://doi.org/10.1145/3105762.3105770

Sudhanshu Kumar Semwal. 1993. A Proposal for using ANNs for CG Animation. CC-AI: The fournal for the Integrated Study of Artificial Intelligence, Cognitive Science and Applied Epistemology 10 (1993), 93-106.

Karen Simonyan and Andrew Zisserman. 2015. Very Deep Convolutional Networks for Large-Scale Image Recognition. In 3rd International Conference on Learning Representations, ICLR 2015, San Diego, CA, USA, May 7-9, 2015, Conference Track Proceedings, Yoshua Bengio and Yann LeCun (Eds.). http://arxiv.org/abs/1409.1556

Greg Turk and Marc Levoy. 1994. Stanford Bunny. http://graphics.stanford.edu/data/ 3Dscanrep/.

Ashish Vaswani, Noam Shazeer, Niki Parmar, Jakob Uszkoreit, Llion Jones, Aidan N Gomez, $€$ ukasz Kaiser, and Illia Polosukhin. 2017. Attention is All you Need. In Advances in Neural Information Processing Systems 30, I. Guyon, U. V. Luxburg, S. Bengio, H. Wallach, R. Fergus, S. Vishwanathan, and R. Garnett (Eds.). Curran Associates, Inc., 5998-6008. http://papers.nips.cc/paper/7181-attention-is-all-youneed.pdf

T. Xu, P. Zhang, O. Huang, H. Zhang, Z. Gan, X. Huang, and X. He. 2018. AttnGAN: Fine-Grained Text to Image Generation with Attentional Generative Adversarial Networks. In 2018 IEEE/CVF Conference on Computer Vision and Pattern Recognition. 1316-1324. https://doi.org/10.1109/CVPR.2018.00143

Y. Zhou, H. Dong, and A. El Saddik. 2020. Deep Learning in Next-Frame Prediction: A Benchmark Review. IEEE Access 8 (2020), 69273-69283. https://doi.org/10.1109/ ACCESS.2020.2987281

Yang Zhou, Zhen Zhu, Xiang Bai, Dani Lischinski, Daniel Cohen-Or, and Hui Huang. 2018. Non-Stationary Texture Synthesis by Adversarial Expansion. ACM Trans. Graph. 37, 4, Article 49 (July 2018), 13 pages. https://doi.org/10.1145/3197517. 3201285 